\title{
Optimal Sensor Transmission Energy Allocation for Linear Control Over a Packet Dropping Link with Energy Harvesting
}

\author{
Steffi Knorn and Subhrakanti Dey
}

\begin{abstract}
This paper studies a closed loop linear control system. The sensor computes a state estimate and sends it to the controller/actuator in the receiver block over a randomly fading packet dropping link. The receiver sends an ACK/NACK packet to the transmitter over a link. It is assumed that the transmission energy per packet at the sensor depletes a battery of limited capacity, replenished by an energy harvester. The objective is to design an optimal energy allocation policy and an optimal control policy so that a finite horizon LQG control cost is minimized. It is shown that in case the receiver to sensor feedback channel is free of errors, a separation principle holds. Hence, the optimal LQG controller is linear, the Kalman filter is optimal and the optimal energy allocation policy is obtained via solving a backward dynamic programming equation. In case the feedback channel is erroneous, the separation principle does not hold. In this case, we propose a suboptimal policy where the controller still uses a linear control, and the transmitter minimizes an expected sum of the trace of an "estimated" receiver state estimation error covariance matrix. Simulations are used to illustrate the relative performance of the proposed algorithms and various heuristic algorithms for both the perfect and imperfect feedback cases. It is seen that the dynamic programming based policies outperform the simple heuristic policies by a margin.
\end{abstract}

\section{INTRODUCTION}

Wireless sensors become more powerful, affordable and compact, and are thus used in many areas, [1]-[4]. Sensors are often located in remote places and cannot be connected to reliable power sources. Thus, sensors are often powered by batteries and can only use a limited amount of energy for sensing, processing and communicating information. Hence, the communication links are unreliable and information might be lost in a random manner. It is therefore an important task to study the effects of such unreliable communication channels on filtering and control. An important line of research started with [5] studying a Kalman filter relying on measurements, that are received from the sensor via a packet dropping channel. The authors show that the resulting Kalman filter and its error covariance matrix are time-varying and stochastic. The mean state covariance can be guaranteed to be bounded if the probability of receiving a packet is above a certain lower bound. These results were further extended in [6]-[12] and an overview of the results can be found in [13]. Other researchers studied the performance of the Kalman filter and different energy allocation techniques can be found in [14], [15].

The impact of packet dropping links was also studied for

This work was supported by a Swedish Research Council (VR) grant Dnr: 621-2013-5395.

The authors are with the Department of Engineering Sciences, Uppsala University, Sweden; Email: \{steffi.knorn; subhra.dey\}@angstrom.uu.se the closed loop control problem. For example, [16] studied a closed loop control system with a linear Gaussian quadratic optimal controller. It was shown that the separation principle holds in the presence of data losses between the sensor and the receiver Kalman filter when the sensor receives perfect feedback about the packet loss process and there exists a critical arrival probability below which the resulting optimal controller fails to stabilize the system. [17] extended these results by assuming that the control signal also is transmitted via an unreliable communication channel. If the arrival of the control packet is acknowledged perfectly at the receiving actuator, the separation principle holds and the optimal LQG control is linear. However, if no such channel feedback exists, the separation principle does not hold and the resulting optimal controller is in general nonlinear, [18].

One way to help overcome the limitations of limited battery capacities is to use energy harvesting as sensors are often placed in an environment where energy can be harvested using solar panels, wind mills or other technical devices. The harvested energy can then be used for data transmission or be stored in the battery for future use. In recent years, a number of authors have addressed the problem of transmission energy allocation for optimizing various metrics related to information transmission when the transmitters are equipped with energy harvesting capability. In [19], throughput optimal and mean delay optimal energy allocation policies in a single sensor node are studied. The optimal energy allocation policies that maximize the mutual information of a wireless link were derived in [20]. In [21], the authors investigated an optimal packet scheduling problem for a single-user energy harvesting wireless communication system. Optimal off-line transmission policies with batteries with limited storage capacities are investigated in [22], where a short-term throughput maximization and the related problem of minimization of the transmission completion time for a given amount of data are studied. These results are further generalized in [23], where fading channels and optimal online policies are considered. Estimation of a dynamical system with a packet dropping link under energy harvesting constraints was first studied in [24], which is the most closely related paper to the current work. In this paper, the authors studied a sensor with energy harvesting, which sends its measurements over a packet dropping link to the receiver. Transmission energy allocation policies, that minimize the sum of the expected error covariance in the presence of perfect or imperfect channel feedback, were derived.

This paper extends [24] to the case of a closed loop control 
system with a packet dropping link between the sensor and the controller at the receiver, and a feedback channel that can be prone to intermittent losses. We study the optimal energy allocation policy at the transmitter and the optimal control design at the receiver such that a finite-time horizon LQG control cost is minimized. The "smart" sensor performs state estimation of the observed linear dynamical system and transmits the current state estimate to the receiver/controller unit via a packet dropping link since [25] showned that it is optimal to send estimates, in contrast to sending measurements, in this case. The receiver sends an acknowledgement whether it has received the state estimate to the transmitter. The transmitter at the sensor is equipped with a battery with finite capacity and an energy harvester. Hence, the transmitter can choose how much energy should be used to transmit the current state estimate (limited by the available energy at the battery, which fluctuates randomly due to the stochastic nature of harvested energy). The time varying fading channel gain and the harvested energy amounts are described by independent and identically distributed (i.i.d.) random processes. Since the probability of dropping the packet is time varying, the transmitter is forced to find a tradeoff amongst spending energy to transmit the current state estimate, keeping energy in reserve for future transmissions, as well as reducing energy overflow due to a finite battery capacity. If the feedback channel is perfect, it is shown that the separation principle holds and the optimal controller is linear. The optimal transmission energy allocation policy is obtained solving a finite horizon backward dynamic programming equation. In case the feedback channel is erroneous, the separation principle does not hold. For this case, we propose a suboptimal policy with a linear controller and a suboptimal transmission energy allocation policy minimizing a finite horizon expected sum of the trace of an estimated receiver state estimation error covariance matrix. Both cases are also studied numerically and compared to various other strategies, including (i) where the current measurement instead of the current state estimate is sent, and (ii) with two suboptimal, heuristic policies for transmission energy allocation.

Section II describes the system model. The cases of perfect and imperfect channel feedback are considered in Sections III and IV. Section V describes two suboptimal heuristic energy allocation policies and all policies are compared via numerical studies in Section VI, followed by conclusions in Section VII.

\section{System Model and Problem Formulation}

This section describes the general structure of the system. Sections III and IV describe the cases of perfect and imperfect channel feedback in more detail. A scheme of the system model (described in detail below) can be found in Figure 1.

\section{A. Plant Model and Sensor}

The plant is modeled as a simple linear system with state $x_{k} \in \mathbb{R}^{n}$, process noise $w_{k} \in \mathbb{R}^{n}$ (i.i.d. Gaussian noise with

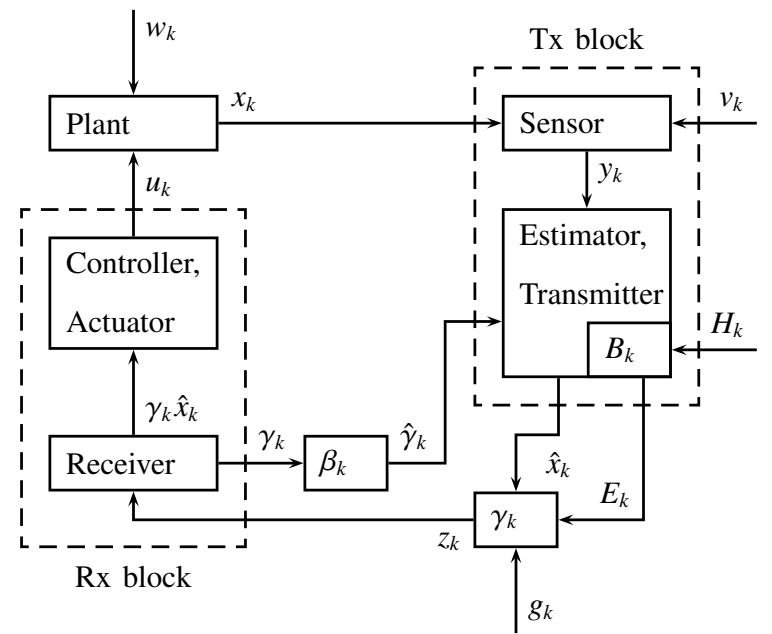

Fig. 1: Scheme of system model when transmitter sends estimates

zero mean and covariance matrix $M=\mathbb{E}\left\{w_{k} w_{k}^{\mathrm{T}}\right\} \geq 0$ ), and a control input $u_{k} \in \mathbb{R}^{p}$, that is $x_{k+1}=A x_{k}+B u_{k}+w_{k}$. The initial state $x_{0}$ is also Gaussian with mean $\bar{x}_{0}$, and covariance $\bar{P}_{0}$, and $A, B$ are matrices with appropriate dimensions.

The sensor produces a noisy measurement of the state $y_{k}=$ $C x_{k}+v_{k}$ where $y_{k} \in \mathbb{R}^{q}$, and $v_{k} \in \mathbb{R}^{q}$ is assumed to be i.i.d. Gaussian noise (independent of $x_{0}$ and $w_{k}$ ) with zero mean and covariance matrix $N=\mathbb{E}\left\{v_{k} v_{k}^{\mathrm{T}}\right\}>0$.

\section{B. State Estimator at the Transmitter}

Assume the sensor is smart with computational capability, and the sensor transmitter forwards a state estimate to the remote estimator/controller. The sensor measurements are used at the transmitter to estimate the current state based on the information set $\mathcal{I}_{k}=\left\{\hat{x}_{0}, y_{l}, \hat{\gamma}_{l-1}: 1 \leq l \leq k\right\}$, where $\hat{\gamma}_{l}$ is the channel feedback acknowledgment, which will be discussed in detail in Section II-E. The estimate is given by

$$
\begin{aligned}
\hat{x}_{k} & :=\hat{x}_{k \mid k}=\mathbb{E}\left\{x_{k} \mid I_{k}\right\} \\
& =\hat{x}_{k \mid k-1}+K_{k}\left(y_{k}-C \hat{x}_{k \mid k-1}\right) \\
\hat{x}_{k+1 \mid k} & =\mathbb{E}\left\{x_{k+1} \mid I_{k}\right\}=A \hat{x}_{k \mid k}+B \hat{u}_{k}
\end{aligned}
$$

where $\hat{u}_{k}$ is the estimated control input which depends on whether the latest channel feedback signal (explained in detail below) has been received or not. The matrix $K_{k}$ should be chosen such that it minimizes the error covariance matrix of the state estimation error, which depend on the feedback communication channel. In case the acknowledgements from the receiver regarding whether the transmitted state estimate has been received or not (ACK/NACK), are always received perfectly, the error covariance matrices follow the standard Riccati difference equations, discussed further in Section III. However, in case the ACK/NACK feedback is dropped intermittently, the separation principle no longer holds. This leads to a non-standard form of the error covariance matrices, see Section IV. 


\section{Energy Harvester and Battery Dynamics}

The transmitter has a rechargeable battery equipped with an energy harvester, that can gather energy from the environment. The amount of energy available to be harvested at time slot $k$, denoted by $H_{k}$ and modeled by an i.i.d. process, is stored in the battery and can be used for data transmission. We assume that the energy used for computational purposes at the transmitter are negligible compared to the amount of energy required for transmission. This is particularly true if data is transmitted over a wireless channel to a receiver that is a long distance away. The amount of stored energy in the battery at time $k, B_{k}$, evolves according to

$$
B_{k+1}=\min \left\{B_{k}-E_{k}+H_{k+1} ; \quad \bar{B}\right\}
$$

with $0 \leq B_{0} \leq \bar{B}$ and where $\bar{B}$ is the battery capacity, and $E_{k}$ is the energy used for transmission during the $k$-th slot.

\section{Forward Communication Channel}

A wireless, packet dropping communication channel is used to transmit the state estimate $\hat{x}_{k}$ to the receiver such that the estimate is either exactly received $\left(\gamma_{k}=1\right)$ or completely lost due to corrupted data or substantial delay $\left(\gamma_{k}=0\right)$, where $\gamma_{k}$ is the Bernoulli random variable modeling the packet loss process. The received signal is $z_{k}=\gamma_{k} \hat{x}_{k}$. The probability of successfully transmitting the packet is

$$
\mathbb{P}\left(\gamma_{k}=1 \mid g_{k}, E_{k}\right):=h\left(g_{k} E_{k}\right)
$$

where $g_{k}$ is the time-varying wireless fading channel gain and $E_{k}$ is the transmission energy for transmitting the packet at $k . h:[0, \infty] \rightarrow[0,1]$ is monotonically increasing and continuous.

We assume that the channel gain $g_{k}$ is described by an i.i.d. process, independent of the energy harvesting process $H_{k}$, and known to the transmitter. Based on the channel gain $g_{k}$, and the current battery level $B_{k}$, the transmitter finds an optimal energy allocation policy $\left\{E_{k}\right\}$ in order to minimize a suitable finite horizon control cost. The details of this optimal energy allocation scheme will be provided later in this section.

\section{E. Erroneous Feedback Communication Channel}

After receiving $z_{k}$, the receiver sends an acknowledgment to the transmitter via a packet dropping feedback channel such that the received acknowledgement is of the form

$$
\hat{\gamma}_{k}= \begin{cases}\gamma_{k} & \text { if } \beta_{k}=1 \\ 2 & \text { if } \beta_{k}=0\end{cases}
$$

where the $\beta_{k}$ is a Bernoulli random variable indicating if the ACK/NACK packet has been received with $\mathbb{P}\left(\beta_{k}=0\right)=$ $\eta \in[0,1]$. In case no ACK/NACK is received, the transmitter receives the feedback signal $\hat{\gamma}_{k}=2$ indicating the packet drop.

\section{F. Estimator/Controller and Actuator in the Receiver block}

The controller in the receiver block has access to the information set $\mathcal{I}_{k}^{\mathrm{c}}:=\left\{\hat{x}_{0}^{\mathrm{c}}, z_{l}, \gamma_{l}: 1 \leq l \leq k\right\}$. Since the estimates from the transmitter are dropped intermittently, the state estimate at the Rx block, $\hat{x}_{k}^{\mathrm{c}}=E\left[x_{k} \mid \mathcal{I}_{k}^{\mathrm{c}}\right]$, is

$$
\hat{x}_{k}^{\mathrm{c}}=\gamma_{k} \hat{x}_{k}+\left(1-\gamma_{k}\right)\left(A \hat{x}_{k-1}^{\mathrm{c}}+B u_{k-1}\right) \text {. }
$$

The task of the controller is to design an optimal control sequence $\left\{u_{k}\right\}$ based on the information pattern $\mathcal{I}_{k}^{\mathrm{c}}$ such that a suitable finite horizon control cost is minimized. It is assumed that the link between the Rx block and the plant is lossless, such that the correct control signal $u_{k}$ is applied to the plant.

\section{G. Optimal Transmission Energy and Control Policy Design}

The aim is to find the optimal transmission energy allocation policy $\mathbf{E}^{\mathbf{N}-\mathbf{1}^{*}}$ and the optimal control policy $\mathbf{u}^{\mathbf{N}-\mathbf{1}^{*}}$, that jointly minimize the finite horizon LQG control cost

$$
J\left(\mathbf{u}^{\mathbf{N}-1}, \mathbf{E}^{\mathbf{N}-1}, \bar{x}_{0}, \bar{P}_{0}\right)=\mathbb{E}\left\{x_{N}^{\mathrm{T}} Q x_{N}+\sum_{k=0}^{N-1}\left(x_{k}^{\mathrm{T}} Q x_{k}+u_{k}^{\mathrm{T}} S u_{k}\right)\right\}
$$

where $\mathbf{u}^{\mathbf{N}-\mathbf{1}}=\left\{u_{0}, u_{1}, \ldots, u_{N-1}\right\}$, and $\mathbf{E}^{\mathbf{N}-\mathbf{1}}=$ $\left\{E_{0}, E_{1}, \ldots, E_{N-1}\right\}$, and the dependence of the cost on the mean and the variance of the initial state is explicitly shown. We will show in Section III, that if the feedback channel is perfect, the separation principle holds and the design of the optimal control input $u_{k}$ and the optimal transmission energy $E_{k}$ can be separated. However, in the case of imperfect channel feedback, the optimal choices of $u_{k}$ and $E_{k}$ depend on each other, as shown in Section IV.

Remark 1: The joint optimization of the transmission energy allocation and the control policy can be done at the receiver block if the sensor battery level is known at the receiver, and the optimal energy allocation policy can be fed back to the transmitter. However, this is difficult as this information needs to be transmitted wirelessly and may be lost. Therefore we focus on the scenario where the transmitter designs the optimal energy allocation policy and the receiver designs the optimal control policy. It will be seen below that in the case of perfect channel feedback, the transmitter can design the optimal energy allocation policy due to the separation principle. In the case of imperfect channel feedback, the energy allocation policy design at the transmitter becomes strictly suboptimal.

\section{Perfect Feedback}

In case the channel feedback link is perfect, the control of the closed loop system follows well known principles. After clarifying the dynamics of the error covariance matrices at the estimator and the controller, it will be shown that the separation principle holds. (Since we only consider a finite horizon problem, the quadratic control cost (7) is always bounded and a stability proof is not needed.)

\section{A. Error Covariance Matrices at the Transmitter and Re- ceiver}

The estimator at the Tx block calculates an estimate of the system state via a Kalman filter based on the information set $I_{k}:=\left\{\hat{x}_{0}, y_{l}, \gamma_{l-1}: 1 \leq l \leq k\right\}$. The estimate is given 
in (2). Since it is assumed that the acknowledgements are received without faults, the estimator has perfect knowledge of the state estimate at the controller and hence the applied control input, $\hat{u}_{k}=u_{k}$. The error covariance matrices at the transmitter are

$$
\begin{aligned}
P_{k \mid k} & =\mathbb{E}\left\{\left(x_{k}-\hat{x}_{k \mid k}\right)\left(x_{k}-\hat{x}_{k \mid k}\right)^{\mathrm{T}} \mid \mathcal{I}_{k}\right\}, \\
P_{k+1} & :=P_{k+1 \mid k}=\mathbb{E}\left\{\left(x_{k+1}-\hat{x}_{k+1 \mid k}\right)\left(x_{k+1}-\hat{x}_{k+1 \mid k}\right)^{\mathrm{T}} \mid \mathcal{I}_{k}\right\} .
\end{aligned}
$$

With $e_{k \mid k}=x_{k}-\hat{x}_{k \mid k}$ and $e_{k+1 \mid k}=x_{k+1}-\hat{x}_{k+1 \mid k}=A e_{k \mid k}+w_{k}$, this yields

$$
P_{k+1}=\mathbb{E}\left\{\left(A e_{k \mid k}+w_{k}\right)\left(A e_{k \mid k}+w_{k}\right)^{\mathrm{T}}\right\}=A P_{k \mid k} A^{\mathrm{T}}+M .
$$

Further, choosing $K_{k}=P_{k \mid k-1} C^{\mathrm{T}}\left(C P_{k \mid k-1} C^{\mathrm{T}}+N\right)^{-1}$ leads to the minimal error covariance matrix after updating the estimate $P_{k \mid k}$ in the standard form

$$
P_{k \mid k}=P_{k-1}-P_{k-1} C^{\mathrm{T}}\left(C P_{k-1} C^{\mathrm{T}}+N\right)^{-1} C P_{k-1} .
$$

The initial covariance matrix is given by $P_{0}=\bar{P}_{0}$. Since the current state estimate is intermittently unavailable at the $\mathrm{Rx}$ block, it is replaced by (6). The corresponding estimation error covariance matrix $P_{k}^{\mathrm{c}}:=\mathbb{E}\left\{\left(x_{k}-\hat{x}_{k}^{\mathrm{c}}\right)\left(x_{k}-\hat{x}_{k}^{\mathrm{c}}\right)^{\mathrm{T}} \mid \mathcal{I}_{k}^{\mathrm{c}}\right\}$ is

$$
P_{k}^{\mathrm{c}}=\gamma_{k} P_{k}+\left(1-\gamma_{k}\right)\left(A P_{k-1}^{\mathrm{c}} A^{\mathrm{T}}+M\right) \text {. }
$$

For simplicity, we assume $P_{0}^{\mathrm{c}}:=\bar{P}_{0}$.

\section{B. Separation Principle}

In case the acknowledgements are received without error, the control input $u_{k}$ is perfectly known at the transmitter. Hence, the control signal estimate $\hat{u}_{k}$ in (2) is replaced by $u_{k}$. Thus, the estimation error is independent of the control input as can be verified in (10) (see also [13]). Clearly, the separation principle holds in this case. This implies, that the tasks of obtaining the optimal Kalman filtered state estimate $\hat{x}_{k}, \hat{x}_{k}^{\mathrm{c}}$, calculating the optimal control input $u_{k}^{*}$ at the controller, and computing the optimal energy allocation $E_{k}^{*}$ at the transmitter can be carried out separately. Consequently, implementing the Kalman filters as discussed above is optimal.

\section{LQG Controller}

Since the separation principle holds, the control cost (7) can be minimized by solely optimizing over $u_{k}$ while keeping $E_{k}$ fixed. Further, the optimal controller is linear and has the form

$$
u_{k}^{*}=L_{k} \hat{x}_{k}^{\mathrm{c}}=-\left(B^{\mathrm{T}} G_{k+1} B+S\right)^{-1} B^{\mathrm{T}} G_{k+1} A \hat{x}_{k}^{\mathrm{c}}
$$

with $G_{k}=Q+A^{\mathrm{T}} G_{k+1} A-A^{\mathrm{T}} G_{k+1} B\left(B^{\mathrm{T}} G_{k+1} B+S\right)^{-1} B^{\mathrm{T}} G_{k+1} A$ and the condition $G_{N}=Q$. Details can be found in [13], [26].

\section{Optimal Energy Allocation Policy}

Due to the separation principle, the optimal energy allocation policy at the transmitter can be obtained by minimizing

$$
\min _{0 \leq E_{k} \leq B_{k} \forall k} \sum_{k=0}^{N-1} \mathbb{E}\left\{\operatorname{tr}\left(P_{k}^{\mathrm{c}}\right) \mid E_{k}\right\} .
$$

This problem can be cast as a stochastic control problem where $\left(g_{k}, B_{k}\right)$ forms the state process and $E_{k}$ forms the control action. A finite horizon dynamic programming algorithm can be used to solve the corresponding backward Bellman dynamic programming equation to minimize (14):

$$
V_{k}\left(g_{k}, B_{k}\right)=\min _{0 \leq E_{k} \leq B_{k}}\left\{\operatorname{tr}\left(P_{k}^{\mathrm{c}}\right) \mid E_{k}+\mathbb{E}\left\{V_{k+1}\left(g_{k}, B_{k}\right)\right\}\right\}
$$

with the terminal condition $V_{N}\left(g_{N}, B_{N}\right)=\operatorname{tr}\left(P_{N}^{\mathrm{c}}\right) \mid B_{N}$.

\section{IMPERFECT ACKNOWLEDGEMENTS}

In this section, it will be shown that in case of a packet dropping channel feedback link, the separation principle does not hold. Hence, the optimal energy allocation policy, state estimation algorithm and controller design are not independent of one another.

\section{A. Assumed State Estimate}

Similar to the case of perfect feedback discussed in Section III, the current state estimate at the $\mathrm{Rx}$ block is given by (6). The calculation of the estimate at the Tx block depends on the knowledge of the applied input signal $u_{k}$, which is not directly known by the estimator, but calculated by the controller. Hence, the estimator at the transmitter also has to estimate the current state estimate used by the controller to calculate $u_{k}$ by using the information of the imperfect feedback channel:

$$
\begin{aligned}
\hat{x}_{k}^{\mathrm{ce}}= & \left(1-\beta_{k}\right)\left(h\left(g_{k} E_{k}\right) \hat{x}_{k}+\left(1-h\left(g_{k} E_{k}\right)\right)\left(A \hat{x}_{k-1}^{\mathrm{ce}}+B \hat{u}_{k-1}\right)\right) \\
& +\beta_{k}\left(\gamma_{k} \hat{x}_{k}+\left(1-\gamma_{k}\right)\left(A \hat{x}_{k-1}^{\mathrm{ce}}+B \hat{u}_{k-1}\right)\right) .
\end{aligned}
$$

In case an acknowledgment was received, the information of the acknowledgment is used. Otherwise, the state estimate at the controller is estimated using the package dropping probability of the forward channel.

\section{B. Estimation Error Covariance Matrices and Kalman Filter}

Note that $\hat{u}_{k}$ denotes the assumed control input at the transmitter. For simplicity, it will be assumed that the control input is a stationary policy of the form $u_{k}=L_{k} \hat{x}_{k}^{\mathrm{c}}$. Hence, the term $\hat{u}_{k}$ can be substituted by $L_{k} \hat{x}_{k}^{\text {ce }}$. Since the transmitter only has knowledge of $\hat{u}_{k-1}$ and $\hat{x}_{k}^{\mathrm{ce}}$ instead of $u_{k}$ and $\hat{x}_{k}^{\mathrm{c}}$, the estimation error $e_{k+1 \mid k}=A e_{k \mid k}+B L_{k} e_{k}^{\mathrm{e}}+w_{k}$ now depends on the error $e_{k}^{\mathrm{e}}:=\hat{x}_{k}^{\mathrm{c}}-\hat{x}_{k}^{\mathrm{ce}}$. Defining $P_{k}^{\mathrm{e}}:=\mathbb{E}\left\{e_{k}^{\mathrm{e}}\left(e_{k}^{\mathrm{e}}\right)^{\mathrm{T}}\right\}$ it follows

$$
\begin{aligned}
P_{k+1}= & A P_{k \mid k} A^{\mathrm{T}}+M+B L_{k} P_{k}^{\mathrm{e}} L_{k}^{\mathrm{T}} B^{\mathrm{T}} \\
& +B L_{k} \mathbb{E}\left\{e_{k}^{\mathrm{e}}\left(e_{k \mid k}\right)^{\mathrm{T}}\right\} A^{\mathrm{T}}+A \mathbb{E}\left\{e_{k \mid k}\left(e_{k}^{\mathrm{e}}\right)^{\mathrm{T}}\right\} L_{k}^{\mathrm{T}} B^{\mathrm{T}} .
\end{aligned}
$$

Choosing again $K_{k}=P_{k \mid k-1} C^{\mathrm{T}}\left(C P_{k \mid k-1} C^{\mathrm{T}}+N\right)^{-1}$ in (1) leads to the error covariance matrix after updating the estimate $P_{k \mid k}$ at the estimator as in the perfect feedback case. However, note that the $K_{k}$ in (1) and the error covariance matrices $P_{k+1}$ and $P_{k \mid k}$ now depend on the controller matrix $L_{k}$ and mixed terms such as $\mathbb{E}\left\{e_{k}^{\mathrm{e}}\left(e_{k \mid k}\right)^{\mathrm{T}}\right\}$. 


\section{Suboptimal control design and transmission energy allo- cation}

In case of imperfect acknowledgements the separation principle does not hold since the estimate depends on the controller matrix $L_{k}$. Further, the error covariance matrices $P_{k}, P_{k}^{\mathrm{c}}, P_{k}^{\mathrm{e}}$ and $P_{k}^{\mathrm{ce}}:=\mathbb{E}\left\{e_{k}^{\mathrm{ce}}\left(e_{k}^{\mathrm{ce}}\right)^{\mathrm{T}}\right\}$ depend on each other, hindering an exact analysis. Since the separation principle does not hold, it is not optimal to design the estimator, the LQR controller and the energy allocation policy separately as done in Section III for the case of perfect channel feedback. However, assuming that the probability of dropping the acknowledgement is small, one can use a suboptimal linear controller $u_{k}=L_{k} \hat{x}_{k}^{\mathrm{c}}$, and a suboptimal energy allocation policy at the transmitter by minimizing the $\operatorname{cost} \sum_{k=0}^{N-1} E\left\{\operatorname{tr}\left(P_{k}^{\mathrm{ce}}\right) \mid E_{k}\right\}$. Since $P_{k}^{\mathrm{ce}}$ depends on other error covariance matrices, which are unknown at the transmitter, it is approximated by

$$
\begin{aligned}
P_{k}^{\text {ce }} \approx & \left(\beta_{k} \gamma_{k}+\left(1-\beta_{k}\right) h\left(g_{k} E_{k}\right)\right) P_{k \mid k} \\
& +\left(\beta_{k}\left(1-\gamma_{k}\right)+\left(1-\beta_{k}\right)\left(1-h\left(g_{k} E_{k}\right)\right)\right)\left(A P_{k-1}^{\mathrm{ce}} A^{\mathrm{T}}+M\right)
\end{aligned}
$$

By sacrificing optimality, the computational burden is made much smaller as the optimal nonlinear estimation and control design and the associated optimal energy allocation policies do not have to be solved by dynamic programming. Through numerical results, we see that for small $\eta$, the suboptimal policy performs quite well compared the perfect feedback case.

Remark 2: Note that one can design other suboptimal policies based on additional information such as the current measurement, which can be used to calculate the assumed estimate $\hat{x}_{k}^{\mathrm{ce}}$. The approach here, which is based solely on the known drop-out probability $h\left(g_{k} E_{k}\right)$ is simple to implement. Alternative suboptimal schemes for estimation and control design in a slightly different context can be found in [27]. It is also apparent that a rigorous performance analysis for our suboptimal scheme is difficult, but will be pursued in future work in the context of a long-term average control cost minimization over an infinite horizon.

\section{Heuristic Policies for Energy Allocation}

It is well known that solving the backward dynamic programming equation to determine the optimal energy allocation policy requires a large computational overhead. Hence, it is often desirable to find suboptimal policies, that require much less computational effort.

One very simple suboptimal policy is a "greedy policy" which sets $E_{k}=B_{k}, \forall k$. Hence, at every time step all available energy is used to transmit data regardless of the channel gain.

A second simple heuristic policy is the "inverted channel policy". Assume the required transmission energy such that the expected drop-out probability of the communication channel with channel gain $g_{k}$ is equal to a desired probability $\bar{\gamma}$, is denoted by $E_{\bar{\gamma}}\left(\bar{\gamma}, g_{k}\right)$. Then, the inverted channel energy allocation policy follows the simple rule $E_{k}=\min \left\{B_{k}, E_{\bar{\gamma}}\left(\bar{\gamma}, g_{k}\right)\right\}$.

\section{NuMERICAL EXAMPLES}

In this section, we evaluate the performance of various optimal and suboptimal energy allocation and control design schemes for both perfect and imperfect feedback cases. A scalar system with parameters $A=1.1, B=1, C=1, M=1$, $N=1$ and $P_{x_{0}}=1$ is considered. It is assumed that the sensor uses a binary phase shift keying (BPSK) transmission scheme, [28], with $b=4$ bits per packet. Here, (4) has the form

$$
\mathbb{P}\left(\gamma_{k}=1 \mid g_{k}, E_{k}\right)=h\left(g_{k} E_{k}\right)=\left(\int_{-\infty}^{\sqrt{g_{k} E_{k}}} \frac{1}{\sqrt{2 \pi}} e^{-t^{2} / 2} d t\right)^{b} .
$$

The harvested energy $H_{k}$ and the fading channel gain $g_{k}$ are assumed to be i.i.d. and exponentially distributed with means $\bar{H}=1 \mathrm{mWh}$ and $\bar{g}=1 \mathrm{~dB}$, respectively. The battery capacity is varied between $1 m W h$ and $5 m W h$.

Eight different scenarios have been simulated: In the first and second scenario it is assumed that all channel gains and harvested energies are known a priori (non-causal ('NC') information) and the feedback communication channel is free of errors. In the first scenario, the transmitter sends the current measurement ('send mes.') to the receiver such that the Kalman Filter is computed at the controller/actuator side. In the second scenario, the current state estimate is communicated to the receiver ('send est.'). Although both scenarios are unrealistic as they rely on non-causal information, they constitute an important benchmark to compare the performance of algorithms using causal information. The third and fourth scenario consider the case of causal information (' $\mathrm{C}$ ') and sending measurements comparing the performance of the perfect feedback case ('PF') vs. the imperfect feedback case with $\eta=20 \%$ ('IF'). The fifth and sixth scenarios consider the case of causal information and sending state estimates ('send est.'), again comparing perfect feedback case ('PF') vs. imperfect feedback with $\eta=20 \%$ ('IF'). The greedy policy ('GP') and the inverted channel policy ('IC') with $\bar{\gamma}=0.8$ are used in the seventh and the eighth scenario, respectively.

The example was simulated 48 times using independent randomly generated numbers for the channel gains and harvested energies with the distributions described above and a finite time horizon of $N=25$. In Figure 2, it can be observed that in all cases the average control cost (of the 48 simulations) decreases as the battery capacity grows. Further, sending state estimates clearly outperforms sending measurements. Assuming a feedback dropout probability of $20 \%$ seems to lead to no noticeable change in the performance of the system as the average control cost in case of perfect or imperfect feedback hardly differ. If the feedback dropout probability increases, it is expected that the average control cost will increase compared to its perfect feedback counterpart. It can be further observed that the optimal policies clearly outperform the heuristic policies by a big margin. Hence, investing in solving the dynamic programming algorithm yields a considerable advantage compared to 


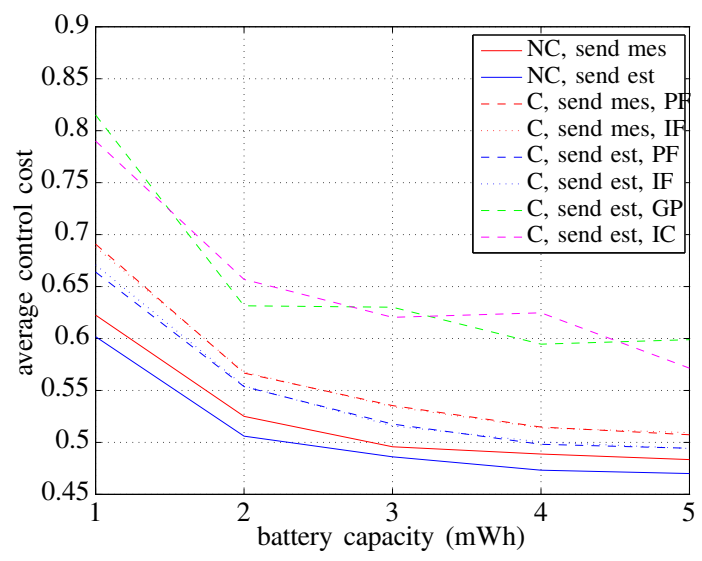

Fig. 2: Average control cost $J$ vs. battery capacity, 'NC' = non-causal information, ' $\mathrm{C}$ ' = causal information, 'mes' = measurements, 'est' = estimates, 'PF' = perfect feedback, 'IF' = imperfect feedback, 'GP' = greedy policy, 'IC' = inverted channel policy

these simple heuristic policies even when feedback channels are imperfect.

\section{Conclusions}

This paper studied a closed loop control system where a sensor runs a local Kalman filter and sends its state estimate to the receiver block consisting of the controller/actuator over a packet dropping link that has a time-varying packet loss probability due to a randomly time-varying fading channel gain. The transmitter is powered by a finite battery and can harvest a random amount of energy from its environment. The receiver sends an ACK/NACK feedback, which may also be lost intermittently. The objective is to design a jointly optimal sensor transmission energy allocation and optimal control design policy for minimizing a finite horizon LQG control cost.

In the case of perfect channel feedback, it is seen that the separation principle holds. Hence, the Kalman filters (at the transmitter and receiver) and a linear controller are optimal and the transmission energy allocation policy that minimizes the sum of the expected estimation error covariance over a finite time horizon can be obtained by standard dynamic programming techniques. In the case of erroneous channel feedback, the separation principle no longer holds. Hence, the optimal estimator and controller design, and the optimal energy allocation policy are in general coupled and nonlinear, and suboptimal designs are presented. Numerical studies are presented illustrating the control cost performance of the various schemes discussed above.

\section{REFERENCES}

[1] I. Akyildiz, W. Su, Y. Sankarasubramaniam, and E. Cayirci, "A survey on sensor networks," IEEE Commun. Mag., vol. 40, no. 8, pp. 102114, 2002.

[2] V. Gungor and G. Hancke, "Industrial wireless sensor networks: Challengesm design, principles and technical approaches," IEEE Trans. Ind. Electron., vol. 56, no. 10, pp. 4258-4265, 2009.
[3] C.-Y. Chong and S. Kumar, "Sensor networks: Evolution, opportunities and challenges," Proc. IEEE, vol. 91, no. 8, pp. 1247-1256, 2003.

[4] V. Gungor, B. Lu, and G. Hancke, "Opportunities and challenges of wireless sensor networks in smart grid," IEEE Trans. Ind. Electron., vol. 57, no. 10, pp. 3557-3564, 2010.

[5] B. Sinopoli, L. Schenato, M. Franceschetti, K. Poolla, M. Jordan, and S. Sastry, "Kalman filtering with intermittent observations," IEEE Trans. Autom. Control, vol. 49, no. 9, pp. 1453-1464, 2004.

[6] X. Liu and A. Goldsmith, "Kalman filtering with partial observation losses," in 43rd IEEE Conference on Decision and Control, 2004, pp. 4180-4186.

[7] Y. Xu and J. Hespanha, "Estimation under uncontrolled and controlled communications in networked control systems," in 44th IEEE Conference on Decision and Control, 2005, pp. 842-847.

[8] M. Huang and S. Dey, "Stability of Kalman filtering with Markovian packet losses," Automatica, vol. 43, no. 4, pp. 698-607, 2007.

[9] M. Epstein, L. Shi, A. Tiwari, and R. Murray, "Probabilistic performance of state estimation across a lossy network," Automatica, vol. 44, no. 12, pp. 3046-3053, 2008.

[10] L. Schenato, "Optimal estimation in networked control systems subject to random delay and packet drop," IEEE Trans. Autom. Control, vol. 53, no. 5, pp. 1311-1317, 2008.

[11] Y. Mo and B. Sinopoli, "A characterization of the critical value for Kalman filtering with intermittent observations," in 47th IEEE Conf. Decision and Control, 2008, pp. 2692-2216.

[12] D. Quevedo, A. Ahlén, A. Leong, and S. Dey, "On Kalman filtering over fading wireless channels with controlled transmission powers," Automatica, vol. 48, no. 7, pp. 1306-1316, 2012.

[13] L. Schenato, B. Sinopoli, M. Franceschetti, K. Poolla, and S. Sastry, "Foundations of control and estimation over lossy networks," Proc. IEEE, vol. 95, no. 1, pp. 163-187, 2007.

[14] D. Quevedo, A. Ahlén, and J. Østergaard, "Energy efficient state estimation with wireless sensors through the use of predictive power control and coding," IEEE Transactions on Signal Processing, vol. 58, no. 9, pp. 4811-4823, 2010.

[15] L. Shi, P. Cheng, and J. Chen, "Sensor data scheduling for optimal state estimation with communication energy constraints," Automatica, vol. 47, no. 8, pp. 1693-1698, 2011.

[16] B. Sinopoli, L. Schenato, M. Franceschetti, K. Poolla, and S. Sastry, "Time varying optimal control with packet losses," in 43rd IEEE Conference on Decision and Control, 2004, pp. 1938-1943.

[17] _ _ "Optimal control with unreliable communication: the TCP case," in American Control Conference, 2005, pp. 3354-3359.

[18] - "LQG control with missing observation and control packets," in 16th IFAC World Congress, 2005.

[19] V. Sharma, U. Mukherji, V. Joseph, and S. Gupta, "Optimal energy managment policies for energy harvesting sensor nodes," IEEE Trans. Wireless Commun., vol. 9, no. 4, 2010.

[20] C. Ho and R. Zhang, "Optimal energy allocation for wireless communications with energy harvesting constraints," IEEE Trans. Signal Process., vol. 60, no. 9, pp. 4808-4818, 2012.

[21] J. Yang, O. Ozel, and S. Ulukus, "Broadcasting with an energy harvesting rechargeable transmitter," IEEE Trans. Wireless Commun., vol. 11, no. 2, pp. 571-583, 2012.

[22] K. Tutuncuoglu and A. Yener, "Optimum transmission policies for battery limited energy harvesting nodes," IEEE Trans. Wireless Commun., vol. 11 , no. 3, pp. 1180-1189, 2012.

[23] O. Ozel, K. Tutuncuoglu, J. Yang, S. Ulukus, and A. Yener, "Transmission with energy harvesting nodes in fading wireless channels: Optimal policies," IEEE J IEEE J. Sel. Areas Commun., vol. 29, no. 8, pp. 1732-1743, 2011.

[24] M. Nourian, A. Leong, and S. Dey, "Optimal energy allocation for Kalman filtering over packet dropping links with imperfect acknowledgments and energy harvesting constraints," IEEE Trans. Autom. Control, vol. 59, no. 8, pp. 2128-2143, 2014.

[25] V. Gupta, B. Hassibi, and R. Murray, "Optimal LQG control across packet-dropping links," System and Control Letters, vol. 56, no. 6, pp. 439-446, 2007.

[26] D. Bertsekas, Dynamic Programming and Optimal Control. Athena Scientific, 1995, vol. 1.

[27] S. Dey, A. Chiuso, and L. Schenato, "Linear encoder-decodercontroller design over channels with packet loss and quantization noise," in European Control Conference, 2015.

[28] J. Proakis, Digital Communications, 4th ed. New York: McGraw-Hill, 2001. 